\title{
The concept of boundedness and the Bohr compactification of a MAP Abelian group
}

\author{
by
}

\author{
Jorge Ga lind o and Salvador Her nán dez (Castellón)
}

\begin{abstract}
Let $G$ be a maximally almost periodic (MAP) Abelian group and let $\mathcal{B}$ be a boundedness on $G$ in the sense of Vilenkin. We study the relations between $\mathcal{B}$ and the Bohr topology of $G$ for some well known groups with boundedness $(G, \mathcal{B})$. As an application, we prove that the Bohr topology of a topological group which is topologically isomorphic to the direct product of a locally convex space and an $\mathcal{L}_{\infty}$-group, contains "many" discrete $C$-embedded subsets which are $C^{*}$-embedded in their Bohr compactification. This result generalizes an analogous theorem of van Douwen for the discrete case and some other ones due to Hartman and Ryll-Nardzewski concerning the existence of $I_{0}$-sets.

We also obtain some results on preservation of compactness for the Bohr topology of several types of MAP Abelian groups, like $\mathcal{L}_{\infty}$-groups, locally convex vector spaces and free Abelian topological groups.
\end{abstract}

1. Introduction. Let $G$ be a maximally almost periodic Abelian (MAPA) group in the sense of von Neumann. That means, in the Abelian case, that for all $e_{G} \neq x \in G$ there is a continuous character $\chi$ such that $\chi(x) \neq 1$. The class of MAPA groups contains most of the relevant families of Abelian topological groups. For instance, locally compact Abelian (LCA) groups, free Abelian topological groups and the additive groups of locally convex vector spaces are MAPA groups.

Let $G$ be an arbitrary Abelian topological group. The set of all continuous characters of $G$, with addition defined pointwise, is an Abelian group again. This group is called the dual group of $G$ and it is denoted by $\widehat{G}$. On $G$ two topologies will usually be considered: its original topology and the topology of pointwise convergence on elements of $\widehat{G}$. The latter is usually called the Bohr topology of $G$ and is denoted by $G^{+}$. The topology of $G^{+}$is a totally

1991 Mathematics Subject Classification: 22A05, 22B05, 46A04, 46A99, 54C45, 54H11.

Key words and phrases: Bohr topology, LCA group, $\mathcal{L}_{\infty}$-group, boundedness, locally convex vector space, $D F$-space, maximally almost periodic, respects compactness, $C$ embedded, $C^{*}$-embedded.

Research partially supported by Spanish DGES grant number PB96-1075. 
bounded group topology, weaker than the original topology of $G$, and the completion of $G^{+}$is $b G$, the Bohr compactification of $G$.

One main problem concerning the Bohr compactification of a topological group is the identification of those properties of $G$ and $G^{+}$that are common to both topologies. It is not known, in general, how the topology of $G^{+}$is placed with respect to the topology of $G$. Our aim in this paper is to confront the families of bounded sets that appear naturally in both $G$ and $G^{+}$. A basic question we are concerned with is when $G$ and $G^{+}$share the same compact subsets. Results in this direction are commonplace in functional analysis, the Banach-Steinhaus theorem being a good example.

More or less, it can be said that the preservation of compact-like properties from $G^{+}$to $G$ involves "uniform boundedness" results and, in many cases, it can be applied to prove the continuity of certain related algebraic homomorphisms.

This was the reason which led Glicksberg to prove the following theorem:

TheOREM 1.1 [10]. Let $G$ be a LCA group. If $K$ is a subset of $G$ which is relatively compact in $G^{+}$, then $K$ is relatively compact in $G$.

As Glicksberg himself points out in [10], "regarding boundedness in that situation as meaning "relatively compact", this theorem is the exact analogue of the uniform boundedness principle for Banach spaces'.

Approaching the problem from a different viewpoint, E. van Douwen [6] considered the Bohr topology of a discrete Abelian group. A subset $A$ of a topological space $X$ is said to be $C$-embedded (respectively $C^{*}$-embedded) if every continuous real-valued function on $A$ (respectively every bounded continuous real-valued function on $A$ ) can be extended to a continuous function on $X$. Then van Douwen's main result can be stated in the following way:

Theorem 1.2 [6, Theorem 1.1.3]. Let $G$ be a discrete Abelian group and let $A$ be an infinite subset of $G$. Then there is a subset $B$ of $A$ with $|B|=|A|$ such that $B$ is discrete and $C^{*}$-embedded in $b G$ and $C$-embedded in $G^{+}$.

In the same paper, van Douwen stated an extension of this theorem to the real line, but left unanswered whether or not the result is true for LCA groups. On this line, Hartman and Ryll-Nardzewski [11] had already obtained the main steps to prove the existence of discrete and $C^{*}$-embedded subsets of $b G$ ( $I_{0}$-sets in their terminology) contained in $G$, for every LCA group $G$.

Van Douwen also applies his theorem to prove that the only convergent sequences in $G_{\mathrm{d}}^{+}$are the eventually constant ones. This fact suggests that a generalization of van Douwen's theorem, which we think is also an interesting goal in itself, will also be a useful tool to handle the generalization of Glicksberg's theorem to bounded subsets of MAPA groups. Therefore, the following two questions are dealt with in this paper. 
Q1. Generalize van Douwen's result to the class of LCA groups and other relevant classes of MAPA groups.

Q2. Extend Glicksberg's result to general MAPA groups and standard families of bounded subsets in them.

To investigate these two questions, the notion of boundedness will be basic. It appears in Chapter 4 of [1] and was introduced by Vilenkin in 1951 in [32] to extend the Pontryagin-van Kampen duality.

To finish this introduction, a few words about notation and terminology. Let $G$ be an Abelian group. The order of $g \in G$ is denoted by $o(g)$. For $A \subseteq G,\langle A\rangle$ denotes the smallest subgroup of $G$ containing $A$. If $G$ is a topological group, then $\mathcal{N}_{0}(G)$ is the set of all open neighbourhoods of the identity. If $\left\{G_{i}\right\}_{i \in I}$ is a collection of Abelian topological groups, $\prod_{i \in I} G_{i}$ is their direct product, and $\bigoplus_{i \in I} G_{i}$ their direct sum (weak direct product). It will usually be assumed that $\prod_{i \in I} G_{i}$ is endowed with the Tikhonov topology and that $\bigoplus_{i \in I} G_{i}$ is endowed with the finest group topology making the canonical inclusion $G_{j} \rightarrow \bigoplus_{i \in I} G_{i}$ continuous for every $j \in I$. By the strict inductive limit of a sequence $\left\{G_{1} \subseteq \ldots \subseteq G_{n} \subseteq \ldots\right\}$ of Abelian topological groups will be meant the group $G=\bigcup_{n<\omega} G_{n}$ endowed with the finest group topology making continuous all the inclusion mappings of $G_{n}$ into $G$.

2. Boundedness. Let $G$ be an Abelian group. According to Vilenkin, a boundedness on $G$ is a family $\mathcal{B}$ of subsets of $G$, called bounded sets, satisfying the following conditions:

1. If $X$ is bounded then so is $-X$.

2. Subsets of bounded sets are bounded.

3. If $X$ and $Y$ are bounded then so are $X \cup Y$ and $X+Y$.

4. Finite sets are bounded.

If a group $G$ is endowed with a boundedness $\mathcal{B}$, then $(G, \mathcal{B})$ is called a group with boundedness.

Examples of groups with boundedness are: the family of all finite subsets of any Abelian group; the family of all precompact subsets of a topological group; the family of all bounded sets (in the usual sense) of the additive group of a topological vector space.

In [13], Hejcman associated with every topological group $G$ the following boundedness $\mathcal{B}$ : a subset $B$ of $G$ is bounded (i.e., $B \in \mathcal{B}$ ) if and only if for every neighbourhood $U$ of the identity in $G$, there exist a finite $F \subset B$ and a natural number $n$ such that

$$
B \subseteq F+U+. . . .+U .
$$

In the sequel it will be assumed that this is the canonical boundedness for every group under consideration. Notice that (as stated by Hejcman himself 
in [13]) this boundedness coincides with the boundedness of precompact subsets for LCA groups, and with the usual boundedness for locally convex spaces.

The concept of boundedness is inherited by subgroups, quotients and direct products and sums in a natural way:

If $\left\{\left(G_{i}, \mathcal{B}_{i}\right): i \in I\right\}$ is a family of groups with boundedness, then their direct product (resp. direct sum) $G$ is endowed with the following boundedness $\prod_{i \in I} \mathcal{B}_{i}$ (resp. $\left.\bigoplus_{i \in I} \mathcal{B}_{i}\right)$ : A subset $X$ of $G$ is bounded if $\pi_{i}(X) \in \mathcal{B}_{i}$ for all $i \in I$ (and resp. $\pi_{i}(X)=\{0\}$ for all but finitely many $i \in I$ ), where $\pi_{i}: G \rightarrow G_{i}$ is the projection.

Given a group with boundedness $(G, \mathcal{B})$ and a subgroup $H$ of $G$, the boundedness induced by $(G, \mathcal{B})$ on $H$ is $\mathcal{B} \cap H$. If $H$ is a closed subgroup of $G$ and $\pi: G \rightarrow G / H$ is the canonical quotient mapping, then the boundedness induced by $(G, \mathcal{B})$ on $G / H$ is $\pi(\mathcal{B})$.

The following definitions, that stem from the theory of rings of continuous functions, are introduced in connection with the notion of boundedness.

For a group with boundedness $(G, \mathcal{B})$ and a subset $A$ of $G$, the boundedcovering number, $B(A)$, of $A$ is defined by

$$
B(A)=\min \left\{|\mathcal{F}|: A=\bigcup_{F \in \mathcal{F}} F \text { and each } F \in \mathcal{F} \text { is bounded }\right\} .
$$

Two subsets $A$ and $B$ of a topological group $G$ are said to be separated if there are two disjoint closed intervals in $\mathbb{T}$, say $I_{0}$ and $I_{1}$, and a character $\chi \in \widehat{G}$ such that $\chi(A) \subseteq I_{0}$ and $\chi(B) \subseteq I_{1}$.

On the other hand, a boundedness $\mathcal{B}$ on $G$ is said to be separated if for every subset $A \subseteq G$ with bounded-covering number $\alpha$, there is a subset $B$ of $A$ with $|B|=\alpha$ such that every subset of $B$ is separated from its complement in $B$. We then also say that the group with boundedness $(G, \mathcal{B})$ is separated.

Next, some useful properties of groups with separated boundedness are stated. The easy proof of the first two of them is omitted.

Proposition 2.1. Let $\left(G, \mathcal{B}_{1}\right)$ and $\left(H, \mathcal{B}_{2}\right)$ be two groups with boundedness such that $\mathcal{B}_{2}$ is separated and let $\alpha: G \rightarrow H$ be a group homomorphism. If $A$ is a subset of $G$ with $B(A) \leq B(\alpha(A))$, then there exists a subset $B$ of $A$ such that $|B|=B(A)$ and any subset of $B$ is separated from its complement in $B$.

Proposition 2.2. Let $\left\{\left(G_{i}, \mathcal{B}_{i}\right): i \in I\right\}$ be a family of groups with boundedness such that every $\mathcal{B}_{i}$ is separated. Consider on $\prod_{i \in I} G_{i}$ the boundedness $\prod_{i \in I} \mathcal{B}_{i}$ described above. If either $I$ is finite or $B\left(\prod_{i \in I} G_{i}\right)=\aleph_{0}$, then the group with boundedness $\left(\prod_{i \in I} G_{i}, \prod_{i \in I} \mathcal{B}_{i}\right)$ is separated.

Proposition 2.3. Let $\left\{\left(G_{i}, \mathcal{B}_{i}\right): i \in I\right\}$ be a family of MAPA groups with boundedness such that every $\mathcal{B}_{i}$ is separated. Suppose in addition that 
$B\left(G_{i}\right) \leq \aleph_{0}$ for all $i \in I$. Then the group with boundedness $(G, \mathcal{B})=$ $\left(\bigoplus_{i \in I} G_{i}, \bigoplus_{i \in I} \mathcal{B}_{i}\right)$ is separated.

Pr o of. Let $A$ be a subset of $G$ with $B(A) \geq \aleph_{0}$. We have to find $B \subseteq A$ with $|B|=B(A)$ such that every subset of $B$ is separated from its complement in $B$.

If $B\left(\pi_{i_{0}}(A)\right)=B(A)$ for some $i_{0}$ in $I$ (here $\pi_{i_{0}}: \bigoplus_{i \in I} G_{i} \rightarrow G_{i_{0}}$ denotes the canonical projection), then we can apply Proposition 2.1. Hence, assume that $B\left(\pi_{i}(A)\right)<B(A)$ for all $i \in I$.

For $\bar{x} \in G$ and $C \subseteq G$, define $\operatorname{supp} \bar{x}=\left\{i \in I: \pi_{i}(\bar{x}) \neq 0\right\}$ and $\operatorname{supp} C=\bigcup\{\operatorname{supp} \bar{x}: \bar{x} \in C\}$. By elementary properties of cardinal numbers and the definition of bounded-covering number, $B(A)=|\operatorname{supp} A|$. Set now

$J_{0}=\left\{i \in I:\right.$ there exists $\bar{x} \in A$ with $i \in \operatorname{supp} \bar{x}$ and $\left.o\left(\pi_{i}(\bar{x})\right) \neq 2\right\}$.

Define

$$
J= \begin{cases}J_{0} & \text { if }\left|J_{0}\right|=|\operatorname{supp} A|=B(A)(\text { case }(1)) \\ \operatorname{supp} A \backslash J_{0} & \text { if }\left|J_{0}\right|<|\operatorname{supp} A|(\text { case }(2))\end{cases}
$$

Now we construct by transfinite induction a subset $B$ of $A$ all of whose subsets are separated by characters from their respective complements.

To begin with, we take an initial ordinal number $\alpha$ equivalent to $|J|$; that is, if $W(\alpha)$ denotes the set of ordinals preceding $\alpha$, then $|W(\alpha)|=|J|$ and $\alpha$ is the smallest ordinal with this property. For the ordinal 0 we take an element of $A$ as follows:

- If (1), take any $\bar{x}_{0} \in A$ such that there is $i_{0} \in \operatorname{supp} \bar{x}_{0}$ with $o\left(\pi_{i_{0}}\left(\bar{x}_{0}\right)\right)$

- If (2), take any $\bar{x}_{0}$ such that there is $i_{0} \in \operatorname{supp} \bar{x}_{0} \backslash J_{0}$, therefore $o\left(\pi_{i_{0}}\left(\bar{x}_{0}\right)\right)=2$.

Now take $\beta \in W(\alpha)$ and suppose that we have already defined a subset $\left\{\bar{x}_{\gamma}: \gamma<\beta\right\} \subseteq A$ such that for every $\gamma<\beta$ :

- If (1), there is $i_{\gamma} \in \operatorname{supp} \bar{x}_{\gamma} \backslash \bigcup\left\{\operatorname{supp} \bar{x}_{\delta}: \delta<\gamma\right\}$ with $o\left(\pi_{i_{\gamma}}\left(\bar{x}_{\gamma}\right)\right) \neq 2$.

- If (2), there is $i_{\gamma} \in \operatorname{supp} \bar{x}_{\gamma} \backslash\left(\bigcup\left\{\operatorname{supp} \bar{x}_{\delta}: \delta<\gamma\right\} \cup J_{0}\right)$, therefore $o\left(\pi_{i_{\gamma}}\left(\bar{x}_{\gamma}\right)\right)=2$.

Since $\alpha$ is an initial ordinal and $\operatorname{supp} \bar{x}$ is a finite subset of $I$ for all $\bar{x} \in G$, it follows that

$$
\left|\bigcup\left\{\operatorname{supp} \bar{x}_{\gamma}: \gamma<\beta\right\}\right|<|W(\alpha)|=|J|=B(A) .
$$

Thus, we can find an index $i_{\beta} \in J$ satisfying:

- If (1), $i_{\beta} \in J \backslash \bigcup\left\{\operatorname{supp} \bar{x}_{\gamma}: \gamma<\beta\right\}$; that is, there is $\bar{x}_{\beta} \in A$ with $i_{\beta} \in \operatorname{supp} \bar{x}_{\beta}$ and $o\left(\pi_{i_{\beta}}\left(\bar{x}_{\beta}\right)\right) \neq 2$.

- If (2), $i_{\beta} \in J \backslash\left(\bigcup\left\{\operatorname{supp} \bar{x}_{\gamma}: \gamma<\beta\right\} \cup J_{0}\right)$; that is, $i_{\beta} \notin J_{0}$ and there is $\bar{x}_{\beta} \in A$ with $i_{\beta} \in \operatorname{supp} \bar{x}_{\beta}$ and, as a consequence, $o\left(\pi_{i_{\beta}}\left(\bar{x}_{\beta}\right)\right)=2$. 
Thus, by transfinite induction, we obtain a subset $B=\left\{\bar{x}_{\beta}: \beta<\alpha\right\}$ such that $|B|=B(A)$ and every $\bar{x}_{\beta}$ satisfies:

- If (1), there is $i_{\beta} \in \operatorname{supp} \bar{x}_{\beta} \backslash \bigcup\left\{\operatorname{supp} \bar{x}_{\gamma}: \gamma<\beta\right\}$ with $o\left(\pi_{i_{\beta}}\left(\bar{x}_{\beta}\right)\right) \neq 2$.

- If (2), there is $i_{\beta} \in \operatorname{supp} \bar{x}_{\beta} \backslash\left(\bigcup\left\{\operatorname{supp} \bar{x}_{\gamma}: \gamma<\beta\right\} \cup J_{0}\right)$. Therefore, $o\left(\pi_{i_{\beta}}\left(\bar{x}_{\beta}\right)\right)=2$.

Finally, it remains to prove that for every $B_{1} \subseteq B$, the sets $B_{1}$ and $B \backslash B_{1}$ are separated by characters.

Indeed, let $I_{a}$ and $I_{b}$ be two disjoint intervals in the one-dimensional torus $\mathbb{T}$ that are centered at -1 and 1 respectively, and have length greater than or equal to one third of the total length of $\mathbb{T}$.

We shall define inductively a family $\left\{\chi^{i_{\gamma}} \in \widehat{G}_{i_{\gamma}}: \gamma \in W(\alpha)\right\}$ such that

$$
\prod_{\delta \leq \gamma} \chi^{i_{\delta}}\left(\pi_{i_{\delta}}\left(\bar{x}_{\gamma}\right)\right) \in I_{\gamma} \quad \text { for all } \gamma<\alpha
$$

To simplify notation, set $J^{\beta}=\left\{\delta: \delta<\beta\right.$ and $\left.i_{\delta} \in \operatorname{supp} \bar{x}_{\beta}\right\}$ for every $\beta<\alpha$. Also, let $I_{\beta}$ denote either $I_{a}$ or $I_{b}$ depending on whether $\bar{x}_{\beta}$ belongs to $B_{1}$ or not.

The first inductive step is simple:

- If (1), consider $\bar{x}_{0} \in B$ and $i_{0} \in \operatorname{supp} \bar{x}_{0}$ as defined previously, that is, with $o\left(\pi_{i_{0}}\left(\bar{x}_{0}\right)\right) \neq 2$. Then it is easily verified (note that the groups under consideration are MAPA) that there is $\chi^{i_{0}} \in \widehat{G}_{i_{0}}$ such that $\chi^{i_{0}}\left(\pi_{i_{0}}\left(\bar{x}_{0}\right)\right) \in I_{0}$.

- If (2), take $\bar{x}_{0} \in B$ and $i_{0} \in \operatorname{supp} \bar{x}_{0} \backslash J_{0}$. Again, there is $\chi^{i_{0}} \in \widehat{G}_{i_{0}}$ such that $\chi^{i_{0}}\left(\pi_{i_{0}}\left(\bar{x}_{0}\right)\right) \in I_{0}$.

Suppose we have already defined $\left\{\chi^{i_{\gamma}} \in \widehat{G}_{i_{\gamma}}: \gamma<\beta\right\}$ satisfying $(*)$, for some $\beta$ in $W(\alpha)$. Since $\operatorname{supp} \bar{x}_{\beta}$ is finite, there is $\gamma_{\beta}<\beta$ such that $\gamma_{\beta} \geq \delta$ for any $\delta \in J^{\beta}$. Then

$$
\prod_{\delta \leq \beta} \chi^{i_{\delta}}\left(\pi_{i_{\delta}}\left(\bar{x}_{\beta}\right)\right)=\chi^{i_{\beta}}\left(\pi_{i_{\beta}}\left(\bar{x}_{\beta}\right)\right) \cdot \prod_{\delta \leq \gamma_{\beta}} \chi^{i_{\delta}}\left(\pi_{i_{\delta}}\left(\bar{x}_{\beta}\right)\right) .
$$

Now, consider $t_{\beta}=\prod_{\delta \leq \gamma_{\beta}} \chi^{i \delta}\left(\pi_{i_{\delta}}\left(\bar{x}_{\beta}\right)\right) \in \mathbb{T}$.

- If (1), since the length of $t_{\beta}^{-1} I_{\beta}$ is again at least one third of that of the torus, it is easy to find $\chi^{i_{\beta}} \in \widehat{G}_{i_{\beta}}$ such that $\chi^{i_{\beta}}\left(\pi_{i_{\beta}}\left(\bar{x}_{\beta}\right)\right) \in t_{\beta}^{-1} I_{\beta}$.

- If (2), since $o\left(\pi_{i_{\delta}}\left(\bar{x}_{\beta}\right)\right)=2$ for every $\delta<\alpha, t_{\beta}$ is necessarily 1 or -1 . So $t_{\beta}^{-1} I_{\beta}$ is again either $I_{a}$ or $I_{b}$, and there is $\chi^{i_{\beta}} \in \widehat{G}_{i_{\beta}}$ such that $\chi^{i_{\beta}}\left(\pi_{i_{\beta}}\left(\bar{x}_{\beta}\right)\right) \in t_{\beta}^{-1} I_{\beta}$.

In either case condition $(*)$ holds for $\beta$. Hence, we have obtained a family $\left\{\chi^{i_{\beta}} \in \widehat{G}_{i_{\beta}}: \beta \in W(\alpha)\right\}$ satisfying $(*)$. 
Now, we define a continuous character $\bar{\chi}=\left(\chi_{i}\right)_{i \in I} \in \prod_{i \in I} \widehat{G}_{i} \subseteq \widehat{G}$ as follows:

$$
\chi_{i}= \begin{cases}0 & \text { if } i \neq i_{\delta} \text { for any } \delta<\alpha, \\ \chi^{i_{\delta}} & \text { if } i=i_{\delta} \text { for some } \delta<\alpha .\end{cases}
$$

Then $\bar{\chi}\left(\bar{x}_{\beta}\right)=\prod_{\delta<\alpha} \chi^{i_{\delta}}\left(\pi_{i_{\delta}}\left(\bar{x}_{\beta}\right)\right)$ for every $\beta<\alpha$. But since $i_{\delta}$ does not belong to $\operatorname{supp} \bar{x}_{\beta}$ unless $\delta \leq \beta$, we obtain

$$
\bar{\chi}\left(\bar{x}_{\beta}\right)=\prod_{\delta \leq \beta} \chi^{i_{\delta}}\left(\pi_{i_{\delta}}\left(\bar{x}_{\beta}\right)\right) \in I_{\beta} \quad \text { for all } \beta<\alpha
$$

and the proof is complete.

The following proposition shows that our canonical boundedness is natural for direct products. The easy proof is omitted.

Proposition 2.4. Let $\left\{\left(G_{i}, \mathcal{B}_{i}\right)\right\}_{i \in I}$ be a collection of topological groups provided with their canonical boundedness and let $G=\prod_{i \in I} G_{i}$. Then the product boundedness $\prod_{i \in I} \mathcal{B}_{i}$ and the canonical boundedness on $G$ coincide.

3. Groups with separated boundedness. In this section it is proved that Abelian $\mathcal{L}_{\infty}$-groups and the additive groups of $D F$-spaces endowed with their canonical boundednesses are separated.

We recall that an $\mathcal{L}_{\infty}$-group is a topological group whose topology is the intersection of a non-increasing sequence of locally compact group topologies. This notion was introduced by Varopoulos in [30] where he made a deep study of these groups, setting the foundations of harmonic analysis on $\mathcal{L}_{\infty}$-groups. Other relevant contributions are the works of Sulley [23] and Venkataraman [31].

We also recall that the $D F$-spaces are a wide class of locally convex spaces containing normed spaces and duals of metrizable spaces, which is closed under most common operations like products, quotients, inductive limits... Every $D F$-space has a fundamental sequence of bounded sets, i.e., a sequence $B_{1} \subset B_{2} \subset \ldots$ of bounded subsets such that every bounded subset is contained in some $B_{k}$. Thus, if $E$ is a $D F$-space it is clear that $B(E)=\aleph_{0}$ for the canonical boundedness on $E$. For a precise definition of $D F$-spaces and their basic properties the reader may consult, for instance, $[16, \S 29.3]$. For our purposes, we just need to quote that, by [16, §29.3(1)], the dual space of a $D F$-space is metrizable and complete.

Let $\mathbb{T}$ denote the one-dimensional torus, now identified with the unit circle of the complex plane. For each $p \in \mathbb{T}$, the set $\left\{x \in \mathbb{T}: x^{n}=p\right\}$ splits the torus into $n$ disjoint connected components of the same length. Clearly, the length of each component can be made arbitrarily small by taking $n$ large enough. 
Now, we prove a series of technical lemmas.

Lemma 3.1. Let $G$ be a locally connected MAPA group and let $A \subseteq \widehat{G}$. Then the following assertions are equivalent:

(a) $A$ is equicontinuous.

(b) There is $V \in \mathcal{N}_{0}(G)$ such that $\bigcup_{a \in A} a(V) \neq \mathbb{T}$.

Proof. It is trivial that (a) implies (b).

Conversely, assume that there is $V \in \mathcal{N}_{0}(G)$ and $p \in \mathbb{T}$ with $p \notin$ $\bigcup_{a \in A} a(V)$. Let $U$ be a neighbourhood of 1 in $\mathbb{T}$ contained in $\left\{e^{2 \pi i t}:|t| \leq\right.$ $1 / 4\}$ such that $p \notin U$. Take $n$ large enough so that the connected component of $\mathbb{T} \backslash\left\{x \in \mathbb{T}: x^{n}=p\right\}$ which contains the identity, $C_{n}(1)$, lies in $U$. Since the mapping $n: G \rightarrow G$ defined by $n(x)=n x$ is a continuous homomorphism, there exists a connected $W \in \mathcal{N}_{0}(G)$ (note that $G$ is locally connected) such that $n(W) \subseteq V$. It follows that, for all $a \in A$,

$$
a(n(W)) \subseteq a(V) \subseteq \mathbb{T} \backslash\{p\} .
$$

Thus, for $x \in W, a(n(x))=(a(x))^{n} \in \mathbb{T} \backslash\{p\}$. That is, $a(W) \subseteq \mathbb{T} \backslash\{x \in$ $\left.\mathbb{T}: x^{n}=p\right\}$. Moreover, $1 \in a(W)$ for all $a \in A$, and $a(W)$ is connected. Hence $a(W) \subseteq C_{n}(1) \subseteq U$ for all $a \in A$. This proves that $A \subseteq\{\chi \in \widehat{G}$ : $\chi(W) \subseteq U\}$, which is to say that $A$ is equicontinuous (see for instance $[19$, Lemma 2.1]).

The following result generalizes a similar result of van Douwen for the real line [6, Theorem 9.2].

Lemma 3.2. Let $G$ be a locally connected MAPA group which is either locally compact or completely metrizable. If $A \subseteq \widehat{G}$ is not equicontinuous, then for any sequence $\left\{\lambda_{k}\right\}_{k=1}^{\infty}$ of positive numbers there is an infinite subset $B=\left\{\chi_{k}\right\}_{k=1}^{\infty} \subseteq A$ such that for any sequence $\left\{I_{k}\right\}_{k=1}^{\infty}$ of intervals in $\mathbb{T}$ with length $l\left(I_{k}\right)=\lambda_{k}$, there is $x \in G$ with $\chi_{k}(x) \in I_{k}$ for all $k<\omega$.

Proof. First we construct by induction a countable family $\left\{B_{k}\right\}_{k=1}^{\infty} \subseteq$ $\mathcal{N}_{0}(G)$ and a sequence $\left\{\chi_{k}\right\}_{k=1}^{\infty} \subseteq A$ such that $B_{k}$ is symmetric, $\chi_{k}\left(B_{k}\right)=\mathbb{T}$, $l\left(\chi_{k-1}\left(2 B_{k}\right)\right)<\lambda_{k}$ and $2 B_{k} \subseteq B_{k-1}$ for all $k<\omega$.

Let $C$ be the component of 0 in $G$. Since $A$ is not equicontinuous and $C$ is an open subset of $G$, there must exist $\chi_{1} \in A$ such that $\chi_{1}(C) \neq\{0\}$. Since $\chi_{1}(C)$ is a non-trivial connected subgroup of $\mathbb{T}, \chi_{1}$ and $B_{1}=G$ can be chosen for the inductive step $k=1$. Assume now that $\left\{B_{1}, \ldots, B_{k}\right\}$ and $\left\{\chi_{1}, \ldots, \chi_{k}\right\}$ satisfying the foregoing conditions have already been found. Then take $B_{k+1} \in \mathcal{N}_{0}(G)$ connected and symmetric with $l\left(\chi_{k}\left(2 B_{k+1}\right)\right)<\lambda_{k}$ and $2 B_{k+1} \subseteq B_{k}$. By Lemma 3.1, $\bigcup_{\chi \in A} \chi\left(B_{k+1}\right)=\mathbb{T}$. Take $\chi_{k+1} \in A$ such that $-1 \in \chi_{k+1}\left(B_{k+1}\right)$. As $B_{k+1}$ is connected and symmetric, it follows that $\chi_{k+1}\left(B_{k+1}\right)=\mathbb{T}$. Thus the inductive construction of $B_{k}$ and $\chi_{k}$ is complete. 
Let now $\left\{I_{k}\right\}_{k=1}^{\infty}$ be a sequence of intervals with $l\left(I_{k}\right)=\lambda_{k}$ and let $t_{k}$ be their middle points.

Again inductively, we will find a sequence $\left\{x_{k}\right\}_{k=1}^{\infty} \subseteq G$ with $x_{k} \in B_{k}$ such that $\chi_{k}\left(x_{1}+\ldots+x_{k}\right)=t_{k}$ and $\chi_{k}\left(x_{1}+\ldots+x_{k}+2 B_{k+1}\right) \subseteq I_{k}$.

For $k=1$, take $x_{1} \in B_{1}=G$ such that $\chi_{1}\left(x_{1}\right)=t_{1}$.

Suppose that $x_{1}, \ldots, x_{k}$ have been found as required. Since $\chi_{k+1}\left(B_{k+1}\right)$ $=\mathbb{T}$, we have $\chi_{k+1}\left(x_{1}+\ldots+x_{k}+B_{k+1}\right)=\mathbb{T}$. Therefore, there exists $x_{k+1} \in$ $B_{k+1}$ such that $\chi_{k+1}\left(x_{1}+\ldots+x_{k}+x_{k+1}\right)=t_{k+1}$. As $B_{k+2}$ is symmetric and $l\left(\chi_{k+1}\left(2 B_{k+2}\right)\right) \leq \lambda_{k+1}$, it follows that $\chi_{k+1}\left(x_{1}+\ldots+x_{k+1}+2 B_{k+2}\right) \subseteq I_{k+1}$.

Finally, let $\left\{C_{k}\right\}_{k=1}^{\infty}$ be the family of subsets of $G$ defined by

$$
C_{k}=x_{1}+\ldots+x_{k}+2 B_{k+1} \text {. }
$$

It is clear that $\chi_{k}\left(C_{k}\right) \subseteq I_{k}$ and $C_{k} \subseteq C_{k-1}$ for all $k<\omega$. Hence, if every $B_{k}$ is chosen either compact, which is possible whenever $G$ is locally compact, or of diameter less than $1 / k$, in case $G$ is metrizable and complete, we have $\bigcap_{k=1}^{\infty} C_{k} \neq \emptyset$. It is now enough to take $x \in \bigcap_{k=1}^{\infty} C_{k}$ to complete the proof.

REMARK 3.3. The preceding lemma can be extended without difficulty to Cech-complete topological groups, but the present formulation is sufficient for our purposes.

It is clear that, for non-locally connected groups, Lemma 3.2 cannot always hold. For example if $G$ is a group containing an element $x$ of order two, then it is impossible to map $x$ homomorphically into any subinterval of $\mathbb{T}$ unless that interval meets 1 or -1 . Nevertheless, when $G$ is a compact group, a variant of Lemma 3.2 is satisfied. Notice that, when $G$ is a compact group, the only equicontinuous subsets of $\widehat{G}$ are the finite ones. Thus, an application of Pontryagin duality carries out the analogy between the following lemma and Lemma 3.2.

Lemma 3.4. Let $G$ be a discrete Abelian group and let $A$ be an infinite subset of $G$. For every pair $I_{0}, I_{1}$ of disjoint closed intervals in $\mathbb{T}$, each containing at least one nth rooth of unity for all $2 \leq n<\omega$, there exists a subset $B$ of $A$ with $|B|=|A|$ such that, for all $\phi \in\{0,1\}^{B}$, there is $\chi \in \widehat{G}$ with $\chi(b) \in I_{\phi(b)}$ for all $b \in B$.

Proof. The idea of the proof is to apply Zorn's Lemma.

First of all, notice that if there is a subset $B_{1}$ of $A$ with $\left|B_{1}\right|=|A|$ such that $B_{1}$ is contained in a finitely generated subgroup $H$ of $G$, then there is $m<\omega$ such that $H \cong \mathbb{Z}^{m} \times F$ with $F$ a finite Abelian group. Thus, since the dual group of $\mathbb{Z}^{m} \times F$ is locally connected, Lemma 3.2 can be applied to $B_{1}$ and $\widehat{H}$ to get a subset $B$ of $B_{1}$ with the required properties.

Hence, from now on, it will be assumed that subsets of cardinality $|A|$ are not included in finitely generated subgroups of $G$. 
Now, for $B \subseteq A$ and $\phi \in\{0,1\}^{B}$ define $N_{\phi}(B):=\{\chi \in \widehat{G}: \chi(b) \in$ $\left.I_{\phi(b)} \forall b \in B\right\}$. Notice that $N_{\phi}(B)$ is always compact. Set

$$
\mathcal{A}:=\left\{B \subseteq A: N_{\phi}(B) \neq \emptyset \text { for all } \phi \in\{0,1\}^{B}\right\} .
$$

The family $\mathcal{A}$ is not empty. Indeed, take $x \in A$ and $\phi \in\{0,1\}^{\{x\}}$. If $o(x)=$ $\infty$, define $\chi(x)$ arbitrarily in $I_{\phi(x)}$ and then extend it to a homomorphism of $G$ into $\mathbb{T}$. If $o(x)=n$, then there exists $t \in I_{\phi(x)}$ such that $t^{n}=1$. Then define $\chi(x)=t$ and extend it to a homomorphism of $G$ into $\mathbb{T}$. In either case, the extended homomorphism belongs to $N_{\phi}(\{x\})$. Therefore $\mathcal{A} \neq \emptyset$.

The set $\mathcal{A}$ can be ordered in the following way: $B_{1} \preceq B_{2}$ iff

1. $B_{1} \subseteq B_{2}$ and

2. $\left(N_{\phi}\left(B_{2}\right)\right)_{\mid B_{1}}=\left(N_{\phi_{\mid B_{1}}}\left(B_{1}\right)\right)_{\mid B_{1}}$ for all $\phi \in\{0,1\}^{B_{2}}$.

The set $\mathcal{A}$ with this order is inductive. Indeed, assume that $\left\{B_{\alpha}: \alpha \in \Lambda\right\}$ is a chain in $\mathcal{A}$ and define $B=\bigcup_{\alpha \in \Lambda} B_{\alpha}$. Given $\phi \in\{0,1\}^{B}$ it is easily verified that $N_{\phi}(B)=\bigcap_{\alpha \in \Lambda} N_{\phi_{\mid B_{\alpha}}}\left(B_{\alpha}\right)$. Thus, it is enough to show that the family $\left\{N_{\phi_{\mid B_{\alpha}}}\left(B_{\alpha}\right): \alpha \in \Lambda\right\}$ has the finite intersection property. Take $\alpha_{1}, \ldots, \alpha_{n}$ in $\Lambda$ with $B_{\alpha_{1}} \preceq \ldots \preceq B_{\alpha_{n}}$. From the way the order has been defined in $\mathcal{A}$, it is clear that $\bigcap_{i=1}^{n} N_{\phi_{\mid B_{\alpha_{i}}}}\left(B_{\alpha_{i}}\right)=N_{\phi_{\mid B_{\alpha_{n}}}}\left(B_{\alpha_{n}}\right) \neq \emptyset$. Hence $B \in \mathcal{A}$ and it remains to check that $B$ is an upper bound of the chain. Let $\alpha_{0} \in \Lambda$ and $\chi \in N_{\phi_{\mid B_{\alpha_{0}}}}\left(B_{\alpha_{0}}\right)$. For every $\alpha \in \Lambda$, define

$$
P_{\alpha}=\left\{\psi \in N_{\phi_{B_{B_{\alpha}}}}\left(B_{\alpha}\right): \psi_{\left.\right|_{B_{\alpha_{0}}}}=\chi_{\left.\right|_{B_{\alpha_{0}}}}\right\} .
$$

Since $\Lambda$ is a chain, the family $\left\{P_{\alpha}\right\}_{\alpha \in \Lambda}$ of compact sets has the finite intersection property. Therefore, we can choose $\psi \in \bigcap_{\alpha \in \Lambda} P_{\alpha}$. Then $\psi \in N_{\phi}(B)$ and $\psi_{\left.\right|_{B_{\alpha_{0}}}}=\chi_{\left.\right|_{B_{\alpha_{0}}}}$, showing that $B_{\alpha_{0}} \preceq B$. Hence, by Zorn's Lemma, there exists a maximal set $B$ in $\mathcal{A}$.

Suppose now that $|B|<|A|$. By the assumption at the beginning of the proof, we can assume that $|\langle B\rangle|<|A|$ and hence that $A$ is not contained in $\langle B\rangle$.

Suppose for the moment that every $a \in A$ has order 2 and consider $a \in A \backslash\langle B\rangle$. Set $B^{\prime}=B \cup\{a\}$. Since $\left\langle B^{\prime}\right\rangle=\langle B\rangle \oplus\langle a\rangle$, it can easily be proved that $B^{\prime} \in \mathcal{A}$ and $B \prec B^{\prime}$, contrary to the maximality of $B$.

Next we consider the general case. For every $x \in A \backslash\langle B\rangle$ we set as above $B_{x}=B \cup\{x\}$. It suffices to find $x$ such that $B_{x} \in \mathcal{A}$ and $B \prec B_{x}$.

Take $\phi \in\{0,1\}^{B_{x}}$. Let $n_{0} x\left(n_{0} \in \mathbb{N} \cup\{0\}\right)$ be a generator of the cyclic group $\langle x\rangle \cap\langle B\rangle$. If $n_{0} \geq 3$ the interval $I_{\phi(x)}$ contains at least one $n_{0}$ th rooth of every element of $\mathbb{T}$, hence we can take $t \in I_{\phi(x)}$ with $t^{n_{0}}=\chi\left(n_{0} x\right)$ and the same can be done if $n_{0}=0$. By defining $\widetilde{\chi}(x)=t$, the homomorphism $\chi_{\mid\langle B\rangle}$ is extended to $\tilde{\chi} \in N_{\phi}\left(B_{x}\right)$. This proves that $N_{\phi}\left(B_{x}\right) \neq \emptyset$ and hence $B \prec B_{x}^{\prime}$. 
Suppose finally that $2 x \in\langle B\rangle$ for every $x \in A$. Consider the quotient group $G /\langle B\rangle$ and the canonical epimorphism $p: G \rightarrow G /\langle B\rangle$. Every element of $p(A)$ has order two, thus by the particular case above there is a subset $B_{1}$ of $p(A)$ with $\left|B_{1}\right|=|p(A)|$ satisfying the desired properties. If we choose for every $b \in B_{1}$ an element $x_{b} \in A$ with $p\left(x_{b}\right)=b$ it is easily checked that these properties are also satisfied by $B_{0}=\left\{x_{b} \in A: b \in B_{1}\right\}$. Since $|\langle B\rangle|<|A|$, we have $|p(A)|=|A|$, hence $\left|B_{0}\right|=|p(A)|=|A|$ and the proof is complete.

In the sequel, the concept of separated boundedness will be studied for a class of groups containing the additive groups of locally convex vector spaces and LCA groups. For vector spaces there is a well known relationship between continuous characters and continuous linear functionals [14, 23.32]: if $G$ is the additive group of a locally convex vector space $E$, then for every $\chi \in \widehat{G}$ there is a unique continuous linear functional $f$ on $E$ such that $\chi(x)=$ $e^{2 \pi i f(x)}$ for all $x \in G$. In addition, this relationship defines a continuous isomorphism $\phi_{E}$ of $E^{\prime}$ onto $\widehat{G}$.

The following lemma will simplify several proofs in the sequel.

LEMMA 3.5. Let $G$ be a MAPA group containing an open subgroup $K=$ $\lim _{n} K_{n}$ topologically isomorphic to the strict inductive limit of the sequence $\left\{K_{n}\right\}_{n=1}^{\infty}$ of compact groups and let $A \subset K$ be a subset not contained in $K_{n}$ for any $n<\omega$. Then there is a continuous homomorphism

$$
\psi: G \rightarrow \bigoplus_{n=1}^{\infty} \mathbb{T}_{n} \quad\left(\text { each } \mathbb{T}_{n} \cong \mathbb{T}\right)
$$

and a subset $A_{1}$ of $A$ such that, for every infinite subset $B \subset A_{1}, \psi(B)$ is not contained in the finite sum $\bigoplus_{n=1}^{m} \mathbb{T}_{n}$ for any $m<\omega$.

Proof. Since each $K_{n}$ is a compact subgroup of $K_{n+1}$, there is an increasing sequence $\left\{m_{n}\right\}_{n=1}^{\infty}$ of positive integers and two sequences $\left\{\chi_{n}\right\}_{n=1}^{\infty}$ $\subseteq \widehat{K}$ and $\left\{x_{n}\right\}_{n=1}^{\infty} \subseteq A$ such that $x_{n} \in K_{m_{n}+1} \backslash K_{m_{n}}, \chi_{n}\left(K_{m_{n}}\right)=\{1\}$ and $\chi_{n}\left(x_{n}\right) \neq 1$.

Define $\psi: K \rightarrow \bigoplus_{n=1}^{\infty} \mathbb{T}_{n}$ (with $\mathbb{T}_{n} \cong \mathbb{T}$ for all $n<\omega$ ) as follows:

$$
\psi(x)=\left(\chi_{1}(x), \chi_{2}(x), \ldots\right) .
$$

This is a well defined group homomorphism, because for all $x \in K$ there is some $n<\omega$ with $x \in K_{m_{n}}$, and consequently $\chi_{m}(x)=1$ for all $m \geq n$. Let us see that $\psi$ is also continuous. Indeed, let $i_{n}: K_{n} \rightarrow K$ be the inclusion. Since $K=\lim _{\longrightarrow} K_{n}$, it suffices to prove that $\psi \circ i_{n}$ is continuous for all $n<\omega$. Choose $m_{p}$ such that $n \leq m_{p}$; then $\left(\psi \circ i_{n}\right)\left(K_{n}\right) \subseteq \bigoplus_{j=1}^{p} \mathbb{T}_{j}=\prod_{j=1}^{p} \mathbb{T}_{j}$, and if $\pi_{j}: \bigoplus_{j=1}^{p} \mathbb{T}_{j} \rightarrow \mathbb{T}_{j}$ denotes the canonical projection, then $\pi_{j} \circ \psi \circ i_{n}=\chi_{j}$. Thus, $\pi_{j} \circ \psi \circ i_{n}$ is continuous for all $j$ with $1 \leq j \leq p$. This implies that $\psi \circ i_{n}$ is continuous for all $n<\omega$, showing the continuity of $\psi$. 
Since $\bigoplus_{n=1}^{\infty} \mathbb{T}_{n}$ is a divisible group, the homomorphism $\psi$ can be extended to $G$ and, being continuous on the open subgroup $K$, the extended homomorphism is also continuous.

If $A_{1}=\left\{x_{n}\right\}_{n=1}^{\infty}$, it is clear from the definition of $\psi$ that no infinite subset of $A_{1}$ is mapped by $\psi$ into a finite sum $\bigoplus_{n=1}^{m} \mathbb{T}_{n}, m<\omega$.

Theorem 3.6. Let $(G, \mathcal{B})$ be a MAPA group with its canonical boundedness. Suppose that $G$ is topologically isomorphic to a group of the form $E \times G_{0}$, where $E$ is a DF-space and $G_{0}$ contains an open subgroup which is a strict inductive limit of compact groups. Then $(G, \mathcal{B})$ is a group with separated boundedness.

Pr o of. By Propositions 2.2 and 2.4, it is enough to show the separability for each factor $E$ and $G_{0}$.

Let $A$ be an unbounded subset of the $D F$-space $E$. We have $B(E)=\aleph_{0}$, as remarked at the beginning of this section. Thus it is required to find a countably infinite subset $B$ of $A$ such that every subset of $B$ is separated by characters from its complement in $B$.

The set $A$ may be considered as a subset of $E^{\prime \prime}$, the bidual topological vector space. Hence, we may apply Lemma 3.2 for $G$ being the additive group of $E^{\prime}$ with its standard topology (of uniform convergence on bounded subsets of $E$ ), which is complete and metrizable. Thus, it is enough to prove that $\phi_{E^{\prime}}(A)$ is not equicontinuous as a set of mappings from $E^{\prime}$ to $\mathbb{T}$.

Suppose that $\phi_{E^{\prime}}(A)$ is equicontinuous. By Lemma 3.1, there exists an absolutely convex closed neighbourhood $V$ of zero in $E^{\prime}$ and $p \in \mathbb{T}$ such that

$$
\bigcup_{a \in A}\left[\phi_{E^{\prime}}(a)\right](V) \subseteq \mathbb{T} \backslash\{p\}
$$

The inclusion above implies that $a(V)$ is properly included in $[0,1)$ for all $a \in A$. Otherwise, $a(V)$ being symmetric and connected, it would contain all the interval $[-1,1]$, and therefore $\phi_{E^{\prime}}(a)(V)=\mathbb{T}$. Thus, $|\langle a, f\rangle| \leq 1$ for all $a \in A$ and $f \in V$. This implies that $A \subseteq V^{\circ}$ (the polar of $V$ ). Hence clearly $A$ is a bounded subset of $E$, contrary to assumption. This finishes the proof for $D F$-spaces.

Now assume that $G$ is a MAPA group containing an open subgroup which is a strict inductive limit of a sequence of compact groups, say $K=\lim _{n} K_{n}$. Again, we consider an unbounded subset $A$ of $G$ and we look for a subset $B$ of $A$ such that $|B|=B(A)$ and every subset of $B$ is separated from its complement in $B$.

Let $p: G \rightarrow G / K$ be the quotient mapping. Since $G / K$ is discrete, $B(p(A))=|p(A)|$ and Lemma 3.4 applies to $p(A)$. Consequently, if $B(A) \leq$ $|p(A)|$, then Proposition 2.1 applied to the map $p$ finishes the proof.

Now assume that $|p(A)|<B(A)$. Since $K$ is a $\sigma$-compact subgroup of $G$, it follows that $|p(A)|$ is finite and $B(A)=\aleph_{0}$. Thus, there exists a coset 
$L$ of $K$ such that $B(A \cap L)=\aleph_{0}$. Take $D=A \cap L$; then there exists $x_{0} \in G$ with $D \subseteq x_{0}+K$. We may assume without loss of generality that $x_{0}$ is the identity of $G$. Obviously, $D$ is not contained in $K_{n}$ for any $n<\omega$ and Lemma 3.5 yields a continuous homomorphism $\psi: G_{0} \rightarrow \bigoplus_{n=1}^{\infty} \mathbb{T}_{n}$ so that $\psi(D)$ is not contained in any finite sum $\bigoplus_{n=1}^{m} \mathbb{T}_{n}$ for $m<\omega$. It is then easily verified that $B(\psi(D))=B(D)=\aleph_{0}$. The proof is completed by applying Proposition 2.3 to $\bigoplus_{n=1}^{\infty} \mathbb{T}_{n}$ and then Proposition 2.1 to $\psi$.

Corollary 3.7. Let $G$ be an Abelian $\mathcal{L}_{\infty}$-group. Then the canonical boundedness on $G$ is separated.

Proof. It can be easily deduced from the structure theorems of [31] and [23] that every Abelian $\mathcal{L}_{\infty}$-group is isomorphic to the direct product $G=E \times G_{0}$, where $E$ is the direct sum of countably many copies of the real line $\mathbb{R}$ and $G_{0}$ contains an open subgroup isomorphic to the strict inductive limit of a sequence of compact Abelian groups.

By the stability properties of $D F$-spaces, $E$ is a $D F$-space. Thus every $\mathcal{L}_{\infty}$-group satisfies the hypothesis of Theorem 3.6 and the corollary follows.

As mentioned above, it may happen that some groups receive more than one boundedness by means of the operations of taking subgroups, quotients, etc., explained in Section 2. In fact, this kind of groups provide examples of groups with a boundedness that is not separated by continuous characters. The next example arises from some facts pointed out in [1, pp. 155 and 162]. It is mainly an adaptation of [29, Theorem 12].

EXAMPLE 3.8. Let $\mathcal{D}=\mathcal{D}(\Omega)$ be the space of test fuctions on $\Omega$, an open subset of the euclidean space $\mathbb{R}^{n}$. In [28] it is proved that $\mathcal{D}$ contains a closed subspace $Q$ such that $\mathcal{D} / Q$ is topologically isomorphic to a dense non-closed subspace of $\mathbb{R}^{\omega}$, the direct product of countably many copies of the real line. On the other hand, $\mathcal{D}$ is, as a locally convex space, the strict inductive limit of its closed subspaces $\mathcal{D}_{n}=\mathcal{D}\left(K_{n}\right)$, where $\left\{K_{n}\right\}_{n=1}^{\infty}$ is a covering of $\Omega$ formed by compact sets. It is well known $[16, \S 19.5 .4]$ that a subset of $\mathcal{D}$ is bounded if and only if it is contained in $\mathcal{D}_{n_{0}}$ for some $n_{0}<\omega$. Now, $\mathcal{D} / Q$ is not complete, hence if $p: \mathcal{D} \rightarrow \mathcal{D} / Q$ is the quotient mapping, then $p\left(\mathcal{D}_{n}\right) \neq p(\mathcal{D})$ and there is a sequence $\left\{x_{k}\right\}_{k=1}^{\infty} \subseteq \mathcal{D} / Q$ and a sequence $\left\{n_{k}\right\}_{k=1}^{\infty}$ of positive integers such that $x_{k} \in p\left(\mathcal{D}_{n_{k+1}}\right) \backslash p\left(\mathcal{D}_{n_{k}}\right)$. Since $\mathcal{D} / Q$ is metrizable, there exists a sequence $\left\{\lambda_{k}\right\}_{k=1}^{\infty}$ of positive numbers such that the set $A=\left\{\lambda_{k} x_{k}\right\}_{k=1}^{\infty}$ is bounded in $\mathcal{D} / Q$. Obviously, $A$ is not contained in $p\left(\mathcal{D}_{n}\right)$ for any $n<\omega$, hence $A$ is not bounded in the boundedness induced by $\mathcal{D}$ on $\mathcal{D} / Q$.

Considering $\mathcal{D} / Q$ as a subspace of $\mathbb{R}^{\omega}$, it follows that $A$ is a bounded subset of $\mathbb{R}^{\omega}$, and hence $\operatorname{cl}_{\mathbb{R}} \omega A$ is a compact subset of $\mathbb{R}^{\omega}$. If the quotient boundedness induced by $\mathcal{D}$ on $\mathcal{D} / Q$ were separated by continuous characters, 
we could apply Corollary 4.3 (which is proved below) to deduce that $A$, being relatively compact, must be bounded, contrary to the choice of $A$.

4. Respecting compactness. The two questions pointed out in the introduction will be answered in this section. First, van Douwen's result for discrete groups is extended to a wider class of groups containing strictly the class of LCA groups. This gives an answer to Q1. Then it is proved how this extension yields two general results concerning the preservation of compactness from the Bohr topology to the original topology of some MAPA groups. This extends Glicksberg's results and provides an answer to Q2.

First, we prove some results that will be needed in the sequel.

LEMMA 4.1. Let $(G, \mathcal{B})$ be a MAPA group with separated boundedness. For every subset $A$ of $G$ there exists $B \subseteq A$ such that $|B|=B(A), B$ is discrete in $G^{+}$and $C^{*}$-embedded in $b G$.

Proof. Let $A$ be a subset of $G$ with $B(A) \geq \aleph_{0}$. By hypothesis the boundedness $\mathcal{B}$ is separated, hence there is $B \subseteq A$ with $|B|=B(A)$ such that every subset of $B$ is separated from its complement in $B$.

Take $b \in B$ and consider the subsets $\{b\}$ and $B \backslash\{b\}$. There are two disjoint intervals in $\mathbb{T}, I_{0}$ and $I_{1}$, and $\chi \in \widehat{G}$ such that $\chi(b) \in I_{0}$ and $\chi(B \backslash\{b\}) \subseteq I_{1}$. Then $\chi^{-1}\left(\mathbb{T} \backslash I_{1}\right)$ is a neighbourhood of $b$ in the Bohr topology of $G$ that meets $B$ exactly in the point $b$. That is, $B$ is discrete in the Bohr topology.

To see that $B$ is $C^{*}$-embedded in $b G$ it is enough to prove that for every pair of disjoint subsets of $B$, say $B_{0}$ and $B_{1}$, there is $f \in C(b G)$ such that $0 \leq f \leq 1, f_{\mid B_{0}}=0$ and $f_{\mid B_{1}}=1$. Since $\mathcal{B}$ is separated there are two disjoint intervals, $I_{0}$, and $I_{1}$ and $\chi \in \widehat{G}$ such that $\chi\left(B_{0}\right) \subseteq I_{0}$ and $\chi\left(B_{1}\right) \subseteq I_{1}$. Let now $g: \mathbb{T} \rightarrow \mathbb{R}$ be defined by

$$
g(t)=\frac{d\left(t, I_{0}\right)}{d\left(t, I_{0}\right)+d\left(t, I_{1}\right)} ;
$$

clearly $g$ is continuous, $0 \leq g \leq 1, g_{\mid I_{0}}=0$ and $g_{\mid I_{1}}=1$. Since every continuous character extends continuously to the Bohr compactification, it can be assumed that $\chi$ is defined on $b G$. Taking $f=g \circ \chi$ completes the proof.

TheOrem 4.2. Let $(G, \mathcal{B})$ be a MAPA group with separated boundedness. If $A \subseteq G$ is unbounded and compact in the Bohr topology, then $|A| \geq 2^{2^{B(A)}}$.

Pr o of. By Lemma 4.1, there exists $B \subseteq A$ such that $|B|=B(A), B$ is discrete and $C^{*}$-embedded in $b G$. Hence $\operatorname{cl}_{b G} B$ is homeomorphic to $\beta B$, the Stone-Čech compactification of $B$. Since $A$ is Bohr-compact, $\mathrm{cl}_{b G} B \subseteq A$, and since $B$ is discrete, $|\beta B|=2^{2^{|B|}}$. Thus, $|A| \geq 2^{2^{B(A)}}$. 
The next corollary follows immediately from the preceding theorem.

Corollary 4.3. Let $(G, \mathcal{B})$ be a MAPA group with separated boundedness such that $|G| \leq \mathbf{c}$. Then every relatively compact subset of $G$ with the Bohr topology is bounded.

REMARK 4.4. Note that Glicksberg's theorem, for the special case of $G=\mathbb{R}^{n}$, is clearly a consequence of Corollary 4.3 above.

It is actually easy to get a common approach and a self-contained proof of Glicksberg's theorem 1.1 in its full generality and of the well known "uniform boundedness principle" for locally convex spaces using the techniques presented above (see [7]).

The following lemma is a part of van Douwen's theorem [6, Thm. 1.1.3], so we omit its proof here. An alternative proof of van Douwen's theorem which is based on the methods of this paper is given in [8].

Lemma 4.5. Let $G$ be a discrete Abelian group and let $A$ be an infinite subset of $G$. Then $A$ has a subset $B$ with $|B|=|A|$ which is discrete and $C$-embedded in $G^{+}$.

A proof of the following lemma can be found in [25].

Lemma 4.6. If $\phi: G \rightarrow H$ is a continuous homomorphism of topological groups, then $\phi: G^{+} \rightarrow H^{+}$is also continuous with respect to the Bohr topologies.

THEOREM 4.7. Let $G$ be a MAPA group topologically isomorphic to a group of the form $E \times G_{0}$, where $E$ is a $D F$-space and $G_{0}$ contains an open subgroup which is the strict inductive limit of a sequence of compact groups. Endow $G$ with its canonical boundedness. Then, for every subset $A \subseteq G$, there exists $B \subseteq A$ with $|B|=B(A)$ such that $B$ is relatively discrete in $G^{+}, C^{*}$-embedded in $b G$ and $C$-embedded in $G^{+}$.

Proof. Let $\pi_{1}: G \rightarrow E$ and $\pi_{2}: G \rightarrow G_{0}$ be the canonical projections.

Suppose first that $B(A)=B\left(\pi_{1}(A)\right)=\aleph_{0}$. Since $E$ is a $D F$-space there is a sequence $\left\{B_{n}\right\}_{n=1}^{\infty}$ of bounded subsets such that every bounded subset of $E$ is contained in some $B_{n}$. For every $n<\omega$ there exists $x_{n} \in \pi_{1}(A) \backslash B_{n}$. The set $C=\left\{x_{n}: n<\omega\right\}$ is a countable subset of $\pi_{1}(A)$ with the property that every infinite subset of $C$ has infinite bounded-covering number. The set $C$ cannot be weakly bounded since it is not bounded. Thus, there is $\phi \in E^{\prime}$ such that $\phi(C)$ is an unbounded subset of the real line.

Therefore there exists a subset $D=\left\{y_{n}\right\}_{n=1}^{\infty} \subseteq C$ such that $\left|\phi\left(y_{n+1}\right)\right|>$ $\left|\phi\left(y_{n}\right)\right|+1$ for every $n<\omega$. Since, by Lemma $4.6, \phi: E^{+} \rightarrow \mathbb{R}^{+}$is continuous it is clear that $D$ is a discrete subset of $E^{+}$, and it is to be shown that it is also $C$-embedded in $E^{+}$. Indeed, let $V_{n}=\left\{x \in E:\left|\phi(x)-\phi\left(y_{n}\right)\right|<1 / 2\right\}$ 
and, for every $n<\omega$, take $f_{n} \in C\left(E^{+}\right)$with $0 \leq f_{n} \leq 1, f_{n}\left(y_{n}\right)=1$ and $f_{n}\left(E \backslash V_{n}\right)=0$. For any $g \in C(D)$, the function $\widetilde{g}=\sum_{n<\omega} g \cdot f_{n}$ provides a continuous extension of $g$ to the whole $E^{+}$.

On the other hand, the group $E$ with its canonical boundedness is separated (see Theorem 3.6). Lemma 4.1 then implies that there is an infinite subset $B_{1} \subseteq D$ which is $C^{*}$-embedded in $b E$. Since $B_{1} \subseteq \pi_{1}(A)$, for every $x \in B_{1}$ we can choose $y(x) \in G_{0}$ such that $(x, y(x)) \in A$. Then $B=\left\{(x, y(x)): x \in B_{1}\right\}$ is a discrete subset of $G^{+}$that is $C$-embedded in $G^{+}$and $C^{*}$-embedded in $b G$.

Assume now that $B(A)>B\left(\pi_{1}(A)\right)$. Let $K=\underline{\lim } K_{n}$ be the open subgroup of $G_{0}$ which is the strict inductive limit of the sequence $\left\{K_{n}\right\}_{n=1}^{\infty}$ of compact groups, and let $p: G_{0} \rightarrow G_{0} / K$ be the quotient mapping.

Assume that $B(A)>\left|\left(p \circ \pi_{2}\right)(A)\right|$. Since $K$ is a $\sigma$-compact open subgroup of $G_{0}$ it follows that $B(A)=B\left(\pi_{2}(A)\right)=\aleph_{0}$ and $\pi_{2}(A)$ meets only finitely many cosets of $K$. Thus, there must be $x_{0}$ in $G_{0}$ such that

$$
\left(\left(x_{0}+K\right) \cap \pi_{2}(A)\right) \backslash\left(x_{0}+K_{n}\right) \neq \emptyset \quad \text { for all } n<\omega .
$$

There is no loss of generality in assuming that $x_{0}$ is the identity, that is, $K \cap$ $\pi_{2}(A)$ is not contained in $K_{n}$ for any $n<\omega$. Lemma 3.5 yields a continuous homomorphism $\psi: G_{0} \rightarrow \bigoplus_{n=1}^{\infty} \mathbb{T}_{n}$ and a subset $A_{1} \subseteq A$ such that neither $\psi\left(\pi_{2}\left(A_{1}\right)\right)$ nor any of its infinite subsets is bounded in $\bigoplus_{n=1}^{\infty} \mathbb{T}_{n}$ (it is not difficult to see that bounded subsets of direct sums are contained in finite partial sums). Since $\left|\bigoplus_{n=1}^{\infty} \mathbb{T}_{n}\right| \leq \mathbf{c}$, Corollary 4.3 implies that $\psi\left(\pi_{2}\left(A_{1}\right)\right)$ is not relatively compact in $\left(\bigoplus_{n=1}^{\infty} \mathbb{T}_{n}\right)^{+}$, which is a $\sigma$-compact group and, a fortiori, a realcompact group. All these facts imply that $\psi\left(\pi_{2}\left(A_{1}\right)\right)$ is not functionally bounded in $\left(\bigoplus_{n=1}^{\infty} \mathbb{T}_{n}\right)^{+}$, and Lemma 4.6 shows that $\pi_{2}\left(A_{1}\right)$ is not functionally bounded in $\left(G_{0}\right)^{+}$either. Now the proof follows exactly as in the case of $E$ with $E^{+}$replaced by $G_{0}^{+}$.

It remains to handle the case where $B(A)=\left|\left(p \circ \pi_{2}\right)(A)\right| \geq \aleph_{0}$.

Set $C=\left(p \circ \pi_{2}\right)(A) \subseteq G_{0} / K$. Since $C$ is an infinite subset of the discrete group $G_{0} / K$, Lemma 4.5 yields a subset $C_{1} \subseteq C$ with $\left|C_{1}\right|=|C|$ which is discrete and $C$-embedded in $\left(G_{0} / K\right)^{+}$. The canonical boundedness of $G_{0} / K$ is separated by characters, thus there is a subset $B_{1} \subseteq C_{1}$ with $\left|B_{1}\right|=\left|C_{1}\right|$ which is $C^{*}$-embedded in $b\left(G_{0} / K\right)$. Clearly $B_{1}$ is also $C$-embedded in $G_{0}^{+} / K$. Now, for every $z \in B_{1}$ take exactly one $x(z) \in E$ and $y(z) \in G_{0}$ such that $\left(\pi_{2} \circ p\right)(x(z), y(z))=z$ and define $B=\left\{(x(z), y(z)) \in G: z \in B_{1}\right\}$. It is easily verified that $B$ has the required properties.

Corollary 4.8. Let $G$ be an Abelian $\mathcal{L}_{\infty}$-group provided with its canonical boundedness. Then every subset $A$ of $G$ has a subset $B$ with $|B|=B(A)$ such that $B$ is relatively discrete and $C$-embedded in $G^{+}$and $C^{*}$-embedded in $b G$. 
Proof. By the structure theorems mentioned in the proof of Corollary 3.7, $G$ is topologically isomorphic to $\left(\bigoplus_{n=1}^{\alpha} \mathbb{R}\right) \times G_{0}$, where $\alpha \leq \aleph_{0}$ and $G_{0}$ contains an open subgroup isomorphic to the strict inductive limit of a sequence of compact Abelian groups. By Theorem 4.7, the result follows.

The preservation of compactness from the Bohr topology of a MAP group (generalizing, therefore, Glicksberg's theorem) has been the object of thorough research, both in Abelian and non-Abelian groups (see [2, 15, 18, 23]). Comfort, Trigos-Arrieta and $\mathrm{Wu}$ [5] made a main contribution by proving

Theorem 4.9 (Comfort, Trigos-Arrieta and Wu). Let $G$ be a LCA group and let $N$ be a closed metrizable subgroup of $b G$. For every subset $A$ of $G$, if $\{a+N: a \in A\}$ is a compact subset of $b G / N$, then $A+(N \cap G)$ is compact in $G$.

Motivated by this result, the authors of [5] defined a MAPA group that strongly respects compactness to be a group which satisfies the conclusion of Theorem 4.9. They also raised the question of characterizing such groups.

The techniques developed above will now be applied to investigate this question.

First, we give an example of a group respecting compactness but which does not strongly respect compactness.

EXAMPLE 4.10. Let $G$ be a non-closed dense subgroup of $\mathbb{T}$ and let $N$ be a closed subgroup of $\mathbb{T}$ such that $N \cap G=\{1\}$. Clearly $G$ respects compactness and $N$ is metrizable. If we consider a sequence $\left\{b_{n}\right\}_{n=1}^{\infty}$ in $G$ converging to some $x$ in $N, x \neq 1$, then $B=\left\{b_{n}: n<\omega\right\} \cup\{1\}$ is a non-compact subset of $G$ with $B+N$ compact in $b G=\mathbb{T}$. Thus, $G$ does not strongly respect compactness.

Now, a series of preliminary lemmas will be proved.

Lemma 4.11. Let $G$ be a MAPA group, $A$ a subset of $G$, and $N$ a subset of $b G$ containing the neutral element such that $A+N$ is compact in $b G$. If $F$ is an arbitrary subset of $A$, then there exists $A_{0} \subseteq A$ with $\left|A_{0}\right| \leq|N|$ such that

$$
\mathrm{cl}_{b G} F \subseteq A_{0}+N+\mathrm{cl}_{G^{+}}(F-F) .
$$

Proof. For any $x \in N$ such that $(A+\{x\}) \cap \operatorname{cl}_{b G} F \neq \emptyset$, we pick exactly one $a_{x} \in A$ with $a_{x}+x \in \operatorname{cl}_{b G} F$. Then we define

$$
A_{0}=\left\{a_{x}: x \in N \text { and }(A+\{x\}) \cap \operatorname{cl}_{b G} F \neq \emptyset\right\} .
$$

Clearly, $A_{0} \subset A$ and $\left|A_{0}\right| \leq|N|$. Take $b \in \operatorname{cl}_{b G} F$. Since cl $b G F \subseteq A+N$, there are $a \in A$ and $y \in N$ with $b=a+y \in \operatorname{cl}_{b G} F$. Set $b^{\prime}=a_{y}+y \in \operatorname{cl}_{b G} F$. Then $b-b^{\prime}=a-a_{y} \in\left(\operatorname{cl}_{b G} F-\operatorname{cl}_{b G} F\right) \cap G$, and thus $b-b^{\prime} \in \operatorname{cl}_{b G}(F-F) \cap G=$ $\mathrm{cl}_{G^{+}}(F-F)$. Hence $b=a_{y}+y+\left(b-b^{\prime}\right) \in A_{0}+N+\mathrm{cl}_{G^{+}}(F-F)$. 
The following lemma permits us to exhibit many MAPA groups that strongly respect compactness.

Lemma 4.12. Let $\left\{\left(G_{i}, \mathcal{B}_{i}\right): i \in I\right\}$ be a family of MAPA groups with separated boundedness such that the cardinality of their Bohr-separable subgroups is less than $2^{\mathbf{c}}$. Assume that $G$ is a subgroup of the product $\prod_{i \in I} G_{i}$ such that the boundedness on $G$ inherited from the product coincides with the boundedness of relatively compact subsets. Then $G$ strongly respects compactness.

Proof. Consider a subset $A$ of $G$ and a closed subgroup $N$ of $b G$ with $|N|<2^{\mathbf{c}}$ (surely, this is the case if $N$ is metrizable) such that $\{a+N: a \in A\}$ is a compact subset of $b G / N$ or, equivalently, $A+N$ is compact in $b G$.

Suppose that $A+(N \cap G)$ is not compact in $G$. Being closed, $A+(N \cap G)$ is not relatively compact in $G$. By hypothesis it cannot be bounded for the boundedness that $G$ inherits from $\prod_{i \in I} G_{i}$. It follows that $\pi_{i}(A+(N \cap G))=$ $A_{i} \notin \mathcal{B}_{i}$ for some $i \in I$.

The boundedness $\mathcal{B}_{i}$ is separated, therefore Lemma 4.1 implies that there exists $C_{i} \subseteq A_{i}$ with $\left|C_{i}\right|=B\left(A_{i}\right) \geq \aleph_{0}$ and $C_{i}$ relatively discrete in $b G_{i}$ and $C^{*}$-embedded in $b G_{i}$. Let $B_{i}$ be a countable subset of $C_{i}$. Clearly $B_{i}$ is also relatively discrete and $C^{*}$-embedded in $b G_{i}$. As a consequence, $\left|\operatorname{cl}_{b G_{i}} B_{i}\right|=$ $\left|\beta B_{i}\right|=2^{\mathbf{c}}$.

On the other hand, let $\pi_{i \mid G}: G \rightarrow G_{i}$ be the restriction of the $i$ th projection and let $b \pi_{i}: b G \rightarrow b G_{i}$ be its continuous extension. It is clear that $b \pi_{i}(A+N)=\pi_{i}(A)+b \pi_{i}(N)$ is compact in $b G_{i}$, i.e., $A_{i}+b \pi_{i}(N)$ is compact in $b G_{i}$. Lemma 4.11 applied to the set $A_{i}+b \pi_{i}(N)$ and the subset $B_{i} \subseteq A_{i}$ shows that there exists $A_{i}^{\prime}$ with $\left|A_{i}^{\prime}\right| \leq\left|b \pi_{i}(N)\right|$ such that

$$
\operatorname{cl}_{b G_{i}} B_{i} \subseteq A_{i}^{\prime}+b \pi_{i}(N)+\mathrm{cl}_{G_{i}^{+}}\left(B_{i}-B_{i}\right) .
$$

But $\mathrm{cl}_{G_{i}^{+}}\left(B_{i}-B_{i}\right)$ is contained in $\mathrm{cl}_{G_{i}^{+}}\left\langle B_{i}\right\rangle$, which is a Bohr-separable subgroup of $G_{i}$ and, by hypothesis, has cardinality less than $2^{\mathbf{c}}$. Thus, each of the subsets in the sum above has cardinality less than $2^{\mathbf{c}}$, yielding that $\left|\mathrm{cl}_{b G_{i}} B_{i}\right|<2^{\mathbf{c}}$. This contradiction completes the proof.

Corollary 4.13. Let $G$ be a MAPA group whose boundedness of relatively compact subsets is separated. If the cardinality of each Bohr-separable subgroup of $G$ is less than $2^{\mathbf{c}}$, then $G$ strongly respects compactness.

The next result is a consequence of Lemma 4.12 for the additive groups of locally convex spaces. Recall that a locally convex space $E$ is called semiMontel if every bounded subset of $E$ is relatively compact.

THEOREM 4.14. Every semi-Montel space strongly respects compactness. 
Proof. Every locally convex space $E$ can be embedded as a subspace of a direct product of Banach spaces $[16, \S 19.9(1)]$. It is well known that the boundedness that $E$ inherits from this product (with norm-boundedness on each factor) is the canonical boundedness of $E$ which, in the case of a semiMontel space, is exactly the boundedness of relatively compact subsets. Now, every separable subgroup of a Banach space is a subgroup of a separable Banach subspace. Closed subspaces of Banach spaces are closed in the Bohr topology (this is an immediate consequence of the Hahn-Banach theorem) and the cardinality of a separable Banach space does not exceed c. So, Lemma 4.12 applies and the result follows.

A locally convex space $E$ is called semireflexive if the canonical linear mapping from $E$ to $E^{\prime \prime}$ is surjective, and reflexive if this map is a topological isomorphism.

Remus and Trigos showed in [21] that every locally convex vector space with weak topology respects compactness. They also characterized the reflexive locally convex spaces that respect compactness as those which are Montel spaces. Since every semireflexive space is a semi-Montel space when considered with its weak topology, and since every Montel space is a semiMontel space $[16, \S 23.3(2)]$, with the aid of Theorem 4.14 we can push the Remus and Trigos-Arrieta results a bit further.

COROLlary 4.15. Let $E$ be a semireflexive locally convex vector space. Then the following assertions are equivalent:

1. E repects compactness.

2. E strongly respects compactness.

3. $E$ is a semi-Montel space.

Next, we prove one of the main results of this section. It generalizes Glicksberg's theorem, Theorem 2.10 of [5] and the main result of [23]. Notice that, since every $\mathcal{L}_{\infty}$-group is nuclear, it also follows directly from [2] that $\mathcal{L}_{\infty}$-groups respect compactness.

THEOREM 4.16. Every $\mathcal{L}_{\infty}$-group strongly respects compactness.

Proof. By the already mentioned structure theorems, $G=E \times G_{0}$, where $G_{0}$ contains an open subgroup which is the inductive limit of a sequence of compact groups and $E$ is topologically isomorphic to $\bigoplus_{n \leq \alpha} \mathbb{R}_{n}$ with $\alpha \leq \aleph_{0}$ and each $\mathbb{R}_{n}$ topologically isomorphic to the real line.

Let $N$ be a closed subgroup of $b G$ of cardinality less than $2^{\mathbf{c}}$, and let $A$ be a subset of $G$ such that $\{a+N: a \in A\}$ is compact in $b G / N$. Let $\pi_{1}: G \rightarrow E$ and $\pi_{2}: G \rightarrow G_{0}$ be the projections, and consider their extensions $b \pi_{1}: b G \rightarrow b E$ and $b \pi_{2}: b G \rightarrow b G_{0}$. Since $A+(N \cap G)$ is closed in $G$, it is enough to prove that $\pi_{1}(A+(N \cap G))$ and $\pi_{2}(A+(N \cap G))$ are relatively compact. 
Since $b \pi_{1}(A+N)$ is compact in $b E$, Theorem 4.14 shows that $\pi_{1}(A)+$ $\left(b \pi_{1}(N) \cap E\right)$ is compact in $E$. Consequently, $\pi_{1}(A+(N \cap G))$ is relatively compact in $E$.

Let $K=\lim K_{n}$ be the relevant open subgroup of $G_{0}$ and let $p: G_{0} \rightarrow$ $G_{0} / K$ and $b p: b G_{0} \rightarrow b\left(G_{0} / K\right)$ be the quotient mapping and its extension.

Let $B=p\left(\pi_{2}(A+(N \cap G))\right)$. If $B$ is an infinite subset of the discrete group $G_{0} / K$, then van Douwen's theorem [6, Thm. 1.1.3] or Theorem 4.7 yield a subset $C \subseteq B$ with $|C|=|B|$ which is discrete in $\left(G_{0} / K\right)^{+}$and $C^{*}$-embedded in $b\left(G_{0} / K\right)$. Once again, it follows that

$$
\left|\mathrm{cl}_{b\left(G_{0} / K\right)} C\right|=2^{2^{|C|}},
$$

whence

$$
\left|\mathrm{cl}_{b\left(G_{0} / K\right)} B\right|=2^{2^{|B|}} .
$$

Now $B \subseteq b p\left(b \pi_{2}(A+N)\right)$ and we know that $A+N$ is compact in $b G$. It follows that $\mathrm{cl}_{b\left(G_{0} / K\right)} B \subseteq b p\left(b \pi_{2}(A+N)\right)$. Consequently, $2^{2^{|B|}} \leq\left|b p\left(b \pi_{2}(A+N)\right)\right| \leq$ $\left|p\left(\pi_{2}(A)\right)+b p\left(b \pi_{2}(N)\right)\right| \leq \max (|B|,|N|)$, which is impossible.

We thus conclude that $p\left(\pi_{2}(A+(N \cap G))\right)$ is finite and it suffices to prove that $D=\pi_{2}(A+(N \cap G))$ is relatively compact. If $D$ is not compact we can find $x_{0} \in G_{0}$ and an increasing sequence $\left\{n_{k}\right\}_{k=1}^{\infty}$ of integers such that $D \cap\left(x_{0}+K_{n_{k+1}}\right) \backslash\left(x_{0}+K_{n_{k}}\right) \neq \emptyset$ for all $k<\omega$.

There is no loss of generality (replace $A$ by $A-x_{0}$ ) in assuming that $x_{0}$ is the identity of $G$. Then $D \cap\left(K \backslash K_{n}\right) \neq \emptyset$ for all $n<\omega$.

Lemma 3.5 gives a mapping $\psi: G_{0} \rightarrow \bigoplus_{n=1}^{\infty} \mathbb{T}_{n}$ so that $\psi(D \cap K)$ is not contained in any finite sum $\bigoplus_{n=1}^{m} \mathbb{T}_{n}$. Let $\phi=\psi \circ \pi_{2}$ and let $b \phi$ : $b G \rightarrow b\left(\bigoplus_{n=1}^{\infty} \mathbb{T}_{n}\right)$ be its Bohr extension. By [30, Proposition 2, p. 474], $\phi(A+(N \cap G))$ is not relatively compact in $\bigoplus_{n=1}^{\infty} \mathbb{T}_{n}$. This implies that $\phi(A)+\left[b \phi(N) \cap \bigoplus_{n=1}^{\infty} \mathbb{T}_{n}\right]$ cannot be relatively compact either.

On the other hand, $\phi(A)+b \phi(N)=b \phi(A+N)$ is compact in $b\left(\bigoplus_{n=1}^{\infty} \mathbb{T}_{n}\right)$. Now, the group $\bigoplus_{n=1}^{\infty} \mathbb{T}_{n}$ provided with the boundedness of relatively compact subsets is separated (Theorem 3.6) and $\left|\bigoplus_{n=1}^{\infty} \mathbb{T}_{n}\right|<2^{\mathbf{c}}$. Corollary 4.13 shows that $\bigoplus_{n=1}^{\infty} \mathbb{T}_{n}$ strongly respects compactness. Thus we have reached a contradiction with the non-relative compactness of $\phi(A)+[b \phi(N) \cap$ $\left.\bigoplus_{n=1}^{\infty} \mathbb{T}_{n}\right]$. This shows that $D=\pi_{2}(A+(N \cap G))$ must be relatively compact and completes the proof.

As far as we know, there is no result concerning preservation of compactness for free Abelian groups. Moreover, there has been little progress in extending the Pontryagin-van Kampen duality theory to these groups (see [17] and [20] for most recent contributions). Our techniques allow handling free Abelian groups. First, some definitions and notation must be introduced.

A topological space $X$ is called a $\mu$-space if every functionally bounded subset is relatively compact. For $X$ a completely regular space, $L(X)$ de- 
notes the free locally convex space associated with $X$, and $A(X)$ is the free Abelian group on $X$. Algebraically, $L(X)$ and $A(X)$ can be seen as the sets of formal linear combinations of elements of $X$ with coefficients in $\mathbb{R}$ and $\mathbb{Z}$ respectively. So, $A(X)$ can be algebraically embedded in $L(X)$. It is proved in [27] (completing an earlier proof of Tkachenko [24]) that this embedding is also topological.

The following lemma is an elementary result about topological groups.

Lemma 4.17. Let $G$ be a topological group and $X$ a compact subset of $G$. Then the subset $\langle X\rangle_{n}$ consisting of all elements in $\langle X\rangle$ obtained by summing at most $n$ elements of $X \cup X^{-1}$ is compact in $G$.

Lemma 4.18. If $X$ is a $\mu$-space, then every weakly bounded subset of $L(X)$ which is also contained in $A(X)$ is relatively compact.

Pro of. Let $B \subseteq A(X) \subseteq L(X)$ be a weakly bounded subset of $L(X)$. By Corollary 2 of [26], there is $M>0$ and a functionally bounded subset $A$ of $X$ such that

$$
B \subseteq\left\{\sum_{x \in X} \lambda_{x} x \in L(X): \sum_{x}\left|\lambda_{x}\right| \leq M, \lambda_{x}=0 \text { for all } x \notin A\right\} .
$$

Now, since $X$ is a $\mu$-space, $\operatorname{cl}_{X} A$ is a compact subset and $B$ is contained in $\left\langle\operatorname{cl}_{X} A\right\rangle \subseteq A(X)$. Furthermore, if $b \in B$ and $b=\sum_{x \in A} \lambda_{x} x$, we have $\sum_{x \in A}\left|\lambda_{x}\right| \leq M$, and since each $\lambda_{x}$ is in $\mathbb{Z}$, it follows that $b \in\left\langle\operatorname{cl}_{X} A\right\rangle_{[M]+1}$ where $[M]$ denotes the integer part of $M$. Thus, $B \subseteq\left\langle\mathrm{cl}_{X} A\right\rangle_{[M]+1}$, which is compact by Lemma 4.17. This proves that $B$ is relatively compact.

THEOREM 4.19. If $X$ is a $\mu$-space, then $A(X)$ strongly respects compactness.

Pr o of. The free locally convex space $L(X)$ can be embedded, as every locally convex space, in the direct product of a family of Banach spaces. As shown in the proof of Lemma 4.14, Bohr-separable subgroups of Banach spaces have cardinality not exceeding c. By Lemma 4.18 the boundedness that $A(X)$ inherits as a subgroup of this product (i.e., the boundedness inherited from $L(X))$ is exactly the boundedness of relatively compact subsets. Then Lemma 4.12 implies that $A(X)$ strongly respects compactness.

COROLlary 4.20. Let $X$ be any completely regular Hausdorff space. Then $A(X)$ respects compactness.

Proof. It is enough to notice that, if $\mu X$ denotes the smallest subspace of $\beta X$ which is a $\mu$-space and contains $X$, then $A(X)$ is a dense subgroup of $A(\mu X)$.

5. Remarks and open questions. As pointed out in the introduction, our approach was suggested in part by the works of Comfort and 
Ross [4] and van Douwen [6]. In fact, the latter contains a long list of open problems concerning the Bohr topology of Abelian groups that fostered the study of Bohr topologies within general topology. However, results related to Theorem 1.1.3 and Lemma 9.2 of [6] had already been studied in commutative harmonic analysis. See for instance the work of Hartman and Ryll-Nardzewski [11, 12, 22], where the related concept of $I_{0}$-set (or set of Hartman and Ryll-Nardzewski) is introduced.

Concerning Theorem 4.16, as well as Theorems 4.14 and 4.19, note that the closed subgroup $N$ of the Bohr compactification involved is not just metrizable, but of cardinality less than $2^{\mathbf{c}}$. So, in absence of the continuum hypothesis, the property we prove is somewhat stronger than strong preservation of compactness.

Concerning separated boundedness, we mention three questions which suggest possible future research.

In Example 4.10 it is shown that there are MAPA groups respecting compactness but not strongly respecting compactness. Nevertheless, our example is a non-complete group. All complete MAPA groups that we know to respect compactness also strongly respect compactness. Our first question is, therefore,

QUESTION 5.1. Are the properties of respecting compactness and strongly respecting compactness equivalent in the realm of complete MAPA groups?

Lemmas 3.2 and 3.4 have been a basic tool in our determination of MAPA groups with separated boundedness. It seems clear that Lemma 3.2 admits generalizations that will permit broadening the scope of Theorem 4.7.

Question 5.2. Extend Lemmas 3.2 and 3.4 to larger families of MAPA groups.

Finally, we think that an adaptation of the ideas worked out in this paper to non-Abelian groups could provide useful tools to study the Bohr topology in that context.

QuESTION 5.3. Find interesting families of non-Abelian groups provided with separated boundedness.

We are indebted to Professor W. W. Comfort for some helpful suggestions and references. We also wish to thank the referee for his/her constructive report. They have helped us to improve parts of this paper.

\section{References}

[1] W. Banaszczyk, Additive Subgroups of Topological Vector Spaces, Lecture Notes in Math. 1466, Springer, Berlin, 1991. 
[2] W. Banaszczyk and E. Martín-Peinador, The Glicksberg Theorem on weakly compact sets for nuclear groups, Ann. New York Acad. Sci. 788 (1996), 34-39.

[3] W. W. Comfort, S. Hernández and F. J. Trigos-Arrieta, Relating a locally compact Abelian group to its Bohr compactification, Adv. Math. 120 (1996), 322344.

[4] W. W. Comfort and K. A. Ross, Topologies induced by groups of characters, Fund. Math. 55 (1964), 283-291.

[5] W. W. Comfort, F. J. Trigos-Arrieta, and T.-S. Wu, The Bohr compactification, modulo a metrizable subgroup, Fund. Math. 143 (1993), 119-136.

[6] E. K. van Douwen, The maximal totally bounded group topology on $G$ and the biggest minimal G-space, for Abelian groups G, Topology Appl. 34 (1990), 69-91.

[7] J. Galindo, Acotaciones y topologías débiles sobre grupos abelianos maximalmente casi periódicos, Ph.D. Thesis, Universidad Jaume I, Castellón, 1997.

[8] J. Galindo and S. Hernández, On a theorem of van Douwen, preprint, 1998.

[9] L. Gillman and M. Jerison, Rings of Continuous Functions, Van Nostrand, New York, 1960.

[10] I. Glicksberg, Uniform boundedness for groups, Canad. J. Math. 14 (1962), 269276.

[11] S. Hartman and C. Ryll-Nardzewski, Almost periodic extensions of functions, Colloq. Math. 12 (1964), 29-39.

[12] —, - Almost periodic extensions of functions II, ibid. 15 (1966), 79-86.

[13] J. Hejcman, Boundedness in uniform spaces and topological groups, Czechoslovak Math. J. 9 (1962), 544-562.

[14] E. Hewitt and K. A. Ross, Abstract Harmonic Analysis, Vol. I, Grundlehren Math. Wiss. 115, Springer, Berlin, 1963.

[15] R. Hughes, Compactness in locally compact groups, Bull. Amer. Math. Soc. 79 (1973), 122-123.

[16] G. Köthe, Topological Vector Spaces, Vol. I, Grundlehren Math. Wiss. 159, Springer, Berlin, 1969.

[17] A. G. Leiderman, S. A. Morris and V. G. Pestov, The free abelian topological group and the free locally convex space on the unit interval, J. London Math. Soc. 56 (1997), 529-538.

[18] M. Moskowitz, Uniform boundedness for non-abelian groups, Math. Proc. Cambridge Philos. Soc. 97 (1985), 107-110.

[19] N. Noble, k-groups and duality, Trans. Amer. Math. Soc. 151 (1970), 551-561.

[20] V. Pestov, Free Abelian topological groups and the Pontryagin-van Kampen duality, Bull. Austral. Math. Soc. 52 (1995), 297-311.

[21] D. Remus and F. J. Trigos-Arrieta, Abelian groups which satisfy Pontryagin duality need not respect compactness, Proc. Amer. Math. Soc. 117 (1993), 11951200 .

[22] C. Ryll-Nardzewski, Concerning almost periodic extensions of functions, Colloq. Math. 12 (1964), 235-237.

[23] L. J. Sulley, On countable inductive limits of locally compact abelian groups, J. London Math. Soc. (2) 5 (1972), 629-637.

[24] M. Tkachenko, Completeness of free Abelian topological groups, Dokl. Akad. Nauk SSSR 269 (1983), 299-303 (in Russian); English transl.: Soviet Math. Dokl. 27 (1983), 341-345.

[25] F. J. Trigos-Arrieta, Continuity, boundedness, connectedness and the Lindelöf property for topological groups, J. Pure Appl. Algebra 70 (1991), 199-210. 
[26] V. V. Uspenskiŭ, On the topology of a free locally convex space, Dokl. Akad. Nauk SSSR 270 (1983), 1334-1337 (in Russian); English transl.: Soviet Math. Dokl. 27 (1983), 781-785.

[27] - Free topological groups of metrizable spaces, Izv. Akad. Nauk SSSR Ser. Mat. 54 (1990), 1295-1319 (in Russian); English transl.: Math. USSR-Izv. 37 (1991), 657-680.

[28] M. Valdivia, The space of distributions $\mathcal{D}^{\prime}$ is not $B_{r}$-complete, Math. Ann. 211 (1974), 145-149.

[29] - The space $\mathcal{D}(\Omega)$ is not $B_{r}$-complete, Ann. Inst. Fourier (Grenoble) 27 (1977), no. 4, 29-43.

[30] N. Varopoulos, Studies in harmonic analysis, Proc. Cambridge Philos. Soc. 60 (1964), 465-516.

[31] R. Venkataraman, Characterization, structure and analysis on abelian $\mathcal{L}_{\infty}$ groups, Monatsh. Math. 100 (1985), 47-66.

[32] N. Ya. Vilenkin, Theory of characters of topological Abelian groups with a given boundedness, Izv. Akad. Nauk SSSR Ser. Mat. 15 (1951), 439-462 (in Russian).

Departamento de Matemáticas

Universidad Jaume I

12071 Castellón, Spain

E-mail: jgalindo@mat.uji.es hernande@mat.uji.es

Received 7 February 1997;

in revised form 27 July 1998 and 20 October 1998 